\title{
COVID-19-associated meningoencephalitis: A care report and literature review
}

\author{
PU LV ${ }^{1}$, FEN PENG ${ }^{2}$, YEQIONG ZHANG ${ }^{3}$, LINWEI ZHANG $^{3}$, NA LI $^{1}$, LILI SUN $^{3}$,

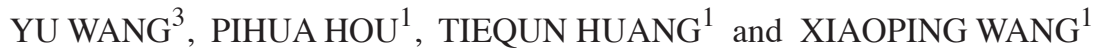 \\ ${ }^{1}$ Department of Healthcare, China-Japan Friendship Hospital, Ministry of Health, Beijing 100029; \\ ${ }^{2}$ Department of Cardiology, Renmin Hospital of Hubei Province, Wuhan, Hubei 430060; ${ }^{3}$ Department of Neurology, \\ China-Japan Friendship Hospital, Ministry of Health, Beijing 100029, P.R. China
}

Received September 1, 2020; Accepted January 8, 2021

DOI: $10.3892 / \mathrm{etm} .2021 .9793$

\begin{abstract}
Infection with severe acute respiratory syndrome coronavirus 2 (SARS-CoV-2) may target the central nervous system and several neurological symptoms have been reported in patients with coronavirus disease 2019 (COVID-19). In the present study, a case of a SARS-CoV-2 complicated with meningoencephalitis was reported. Cerebrospinal fluid (CSF) analyses indicated hyperproteinorrachia but the specimen was negative for SARS-CoV-2 RNA. Furthermore, 10 published articles reporting on patients with COVID-19-associated meningitis/encephalitis were reviewed. Patients diagnosed with COVID-19-associated meningitis/encephalitis had diverse clinical neurological manifestations, including consciousness disturbance, epileptic attacks, psychotic syndrome and meningeal irritation signs. CSF tests revealed elevated protein, lymphocytes and cytokines. SARS-CoV-2 may be detected in the CSF of certain cases. Neuroimaging findings included hyperintense signal changes in the white matter and enhancement of meninges on brain MRI. Certain patients responded well to corticosteroid therapy and had a favorable prognosis, while elderly patients tended to have poor outcomes due to multiple organ dysfunction.
\end{abstract}

\section{Introduction}

Coronavirus disease 2019 (COVID-19), caused by severe acute respiratory syndrome coronavirus 2 (SARS-CoV-2), is characterized by respiratory tract symptoms with potentially severe outcomes. It has been reported by epidemiological studies that COVID-19 presents in the majority of cases with

Correspondence to: Dr Pu Lv or Dr Xiaoping Wang, Department of Healthcare, China-Japan Friendship Hospital, Ministry of Health, 2 Yinghua East Street, Beijing 100029, P.R. China

E-mail:1vpu0505@126.com

E-mail: wang_xpx@163.com

Key words: COVID-19, central nervous system, encephalitis, meningitis upper respiratory symptoms (1). Recently, studies have reported neurological manifestations of COVID-19 in China in up to $36.4 \%$ of hospitalized patients, including alteration of consciousness, headache, dizziness and delirium (2-4). The therapy for COVID-19 remains to be established, although non-pharmacological and preventive treatments have been recommended (5). Like other coronaviruses, SARS-CoV-2 is neurotropic (6). Neurological complications of COVID-19 include cerebrovascular disease, meningitis/encephalitis, acute necrotizing hemorrhagic encephalopathy and Guillain-Barré syndrome (7). However, the clinical characteristics of COVID-19-associated meningitis/encephalitis remain to be defined. Anosmia/dysgeusia has been reported by COVID-19 patient (8-10). This phenomenon provided a hypothesis that COVID-19-associated CNS infection is manifests through a nasopharyngeal route. The present study reported on a patient with COVID-19 presenting with meningio-encephalitis. The clinical characteristics and laboratory results of the patient were investigated. In order to understand the features of COVID-19-associated CNS infection, the literature on COVID-19-associated meningitis/encephalitis cases was reviewed and summarized.

\section{Case report}

Data collection. The patient provided informed written consent for the publication of the present case report. The diagnostic/therapeutic procedures applied were in accordance with institutional and international guidelines for the protection of human subjects. The study was approved by the ethics committee of the China-Japan Friendship Hospital (permit no. 2019-183-K124). Clinical and auxiliary test results were retrieved by the authors. Viral/bacterial detection was performed by real-time reverse transcription-PCR (RT-PCR).

Literature search. Entries related to COVID-19-associated encephalitis/encephalopathy were searched in the PubMed, Chinese National Knowledge Infrastructure (CNKI) and EMBASE databases. Articles that were published between December 2019 and 1st June 2020 were specifically screened to ensure the relevance of the results. The following search terms 
were used: COVID-19, SARS-CoV-2, encephalitis, encephalopathy and meningoencephalitis. A total of 44 articles were retrieved. Case reports or case series that provided detailed clinical information were included. Cohort studies, systematic reviews, meta-analyses and clinical trials were excluded. Of note, no relevant articles were found in the CNKI database. A total of 13 articles met the criteria (11-23). Of these, 1 case of COVID-19 diagnosed with herpes encephalitis (21), 1 study that investigated patients with COVID-19 who did not regain consciousness after withdrawal of invasive mechanical ventilation (23) and another study reporting 2 patients diagnosed with COVID-19 pneumonia complicated by minor neurological symptoms but without neurological imaging or lumbar puncture data (22) were excluded. Thus, a total of 10 articles were finally included in the present study. Of note, due to the rapid spread of the COVID-19 epidemic, other relevant articles may have been published during the preparation of the manuscript of the present study.

\section{Results}

Case presentation. The case was a 90-year-old female of Han Chinese ethnicity who lived in Wuhan city (China) prior to disease onset. She had a history of cerebral lacunar infarction but with no neurological deficits. She was not self-reliant and lived in a health care unit. The patient developed a fluctuatating fever with temperature range between $37.5-38.5^{\circ} \mathrm{C}$, severe cough, sputum, fatigue, chest tightness and shortness of breath on the 1st February 2020. A nasopharyngeal swab was taken and SARS-CoV-2 RNA was detected by RT-PCR (24). The patient had a clear consciousness at admission. The patient's breathing tone was coarse on auscultation and neurological examinations were negative. The blood test results are presented in Table I. The patient presented with elevated CRP and decreased lymphocyte number.

The patient exhibited gradual improvement with absorption of the ground glass opacity on chest CT (Fig. 1A). 25 days after disease onset, the patient suddenly became unconscious and the Glasgow Coma Scale score was determined to be 6 . On neurological examination, the patient was irresponsive to voice and painful stimulation, her pupils were isochoric (2-mm diameter) and reactive, and there was no autonomous activity in any limbs. There were significantly increased muscle tension, positive cortico-spinal tract signs on both sides, a positive meningeal irritation sign, obvious neck stiffness, and a positive Kernig sign. Brain CT was unremarkable compared with a previous brain MRI (Fig. 1B); however, the patient was not subjected to brain MRI at this time-point.

Considering the probable diagnosis of meningoencephalitis, the patient received mannitol and anti-viral therapy (Ganciclovir). A lumbar puncture on March 6th 2020 (10 days after unconsciousness) revealed an opening pressure of $60 \mathrm{mmH}_{2} \mathrm{O}$, cerebrospinal fluid (CSF) cell count with an increased $25 / \mu 1$ (10 mononuclear and 15 polymorphonuclear cells without red blood cells) and CSF protein levels were also increased with $660 \mathrm{mg} / \mathrm{l}$ (normal range: 120-600 mg/l), but normal glucose, chloride and adenosine deaminase levels. Tests for viral/bacterial pathogens (including varicella zoster virus and herpes simplex virus) in the CSF were negative. The result of RT-PCR detection of SARS-CoV-2 RNA in the CSF was negative. However, the patient died of gastrointestinal bleeding 46 days after disease onset.

Summary of clinical characteristics. A total of 10 cases from 10 articles were included in the literature review of the present study. The clinical characteristics of the 10 cases are listed in Table II. The age of the patients ranged from 24 to 75 years and the cases included 6 patients aged $<60$ years. The cases comprised 7 males and 3 females. Only 1 patient had a negative detection result for SARS-CoV-2 RNA in a nasopharyngeal swab. Common neurological signs and symptoms included confusion and coma [patient no. 2 (P2)-5 and P7-9], neck stiffness (P1 and P8), positive meningeal irritation sign (P1, P7), mental disturbance (P1, P2, P4 and P8), seizure (P3 and $\mathrm{P} 6$ ), cognitive impairment (P2 and $\mathrm{P} 4)$, motor dysfunction (P5 and P10) and psychotic symptoms (P3). A total of four patients exhibited abnormalities on brain MRI, which included cerebellar lesions (P5), lesions of the hippocampus in the temporal lobe (P6), cerebral hemorrhage, subarachnoid hemorrhage accompanied by bilateral supratentorial leptomeningeal enhancement (P9) and hyperintensity in the corpus callosum of splenium (P10).

A total of nine cases received lumbar puncture examination, with increased intracranial pressure in only 3 cases (P1, P6 and P7). The CSF protein level was mildly increased in most cases. CSF cytology indicated a slightly increased proportion of white blood cells (WBC) and lymphocytes. Only 3 cases presented with positive SARS-CoV-2 in the CSF (P6, P8 and P9). The patients were negative for other CSF infections and autoimmune antibodies. P2 exhibited increased cytokines (IL-6, IL-8 and TNF- $\alpha$ ) in the CSF and exacerbation clinically. For treatment, corticosteroid therapy and mannitol infusion along with antiviral therapy were administered in some of these 10 cases. Good prognosis and neurological recovery were observed in 6 patients (P1-3 and P7-9), while 2 patients had mild remaining symptoms (P4 and P5). However, elderly patients tended to have poor outcomes and the oldest patient died (P10).

\section{Discussion}

The present study reported on a patient who developed meningoencephalitis after being diagnosed with SARS-CoV-2 infection. The patient gradually recovered from the respiratory symptoms prior to the onset of neurological symptoms. However, the patient suddenly became unconscious and developed neck stiffness and positive cortico-spinal tract signs in all limbs. CSF analysis suggested infection of the central nervous system (CNS) with elevated WBC. A screen for usual pathogens (bacterial, fungal and viral), including SARS-CoV-2, was negative. Based on recently reported diagnostic criteria (25), a possible diagnosis of COVID-19-associated encephalitis was made. Furthermore, published cases of COVID-19-related encephalitis (until 2020 July) were reviewed and their clinical characteristics were summarized. Neurological features mostly started from the time of respiratory symptom onset to 30 days thereafter. The neurological manifestations included irritability, confusion, reduced consciousness and seizures.

As other coronaviruses (7), SARS-CoV-2 is neurotropic. SARS-CoV-2 has been postulated to enter the CNS via 
Table I. Laboratory data of the patient with COVID-19 pneumonia with subsequent meningoencephalitis.

Day of COVID-19 symptoms

\begin{tabular}{|c|c|c|c|c|}
\hline \multirow{2}{*}{ Parameter (normal range) } & & & & \\
\hline & 6 & 20 & 27 & 34 \\
\hline Hemoglobin (g/l, 115-150) & 119 & 119 & 109 & 117 \\
\hline $\operatorname{WBC}\left(10^{9} / 1,3.5-9.5\right)$ & 3.46 & 5.86 & 11.14 & 9.4 \\
\hline Neutrophils $\left(10^{9} / 1,1.8-6.3\right)$ & 1.52 & 4.33 & 10.07 & 8.84 \\
\hline Lymphocytes $\left(10^{9} / 1,1.1-3.2\right)$ & 1.37 & 0.94 & 0.81 & 0.34 \\
\hline Monocytes $\left(10^{9} / 1,0.1-0.6\right)$ & 0.56 & 52 & 0.23 & 0.17 \\
\hline Platelets $\left(10^{9} / 1,125-350\right)$ & 104 & 217 & 225 & 73 \\
\hline Sodium (mmol/1, 133-146) & 138 & 140 & 152 & 146 \\
\hline Potassium (mmol/1, 3.5-5.3) & 3.81 & 3.9 & 3.1 & 2.88 \\
\hline Blood urea nitrogen (mmol/1, 3.1-8.8) & 4 & 3.5 & 9.18 & 7.34 \\
\hline Creatinine (mol/l, 41-81) & 52 & 37 & 37 & 36 \\
\hline Corrected calcium (mmol/1, 2.1-2.37) & 2.47 & 2.49 & & 2.18 \\
\hline $\operatorname{ALT}(\mathrm{U} / 1,7-40)$ & 13 & 11 & & 37 \\
\hline Total bilirubin $(\mu \mathrm{mol} / 1,0-23)$ & 5.8 & 10.3 & & 24 \\
\hline CRP (mg/l, 0.0-10) & 18.2 & 6 & 22.6 & 12 \\
\hline PCT (ng/ml, <0.1) & 0.107 & 0.045 & & 0.316 \\
\hline D-dimer $(\mathrm{ng} / \mathrm{ml},<500)$ & 0.63 & & 1.21 & \\
\hline Creatine kinase (U/l) & 68 & & 25 & 590 \\
\hline TnI (ng/ml, 0-0.04) & & & & 0.382 \\
\hline
\end{tabular}

ALT, alanine transaminase; COVID-19, Coronavirus disease 2019; CRP, C-reactive protein; PCT, procalcitonin; WBC, white blood cell count; TnI, troponin I.

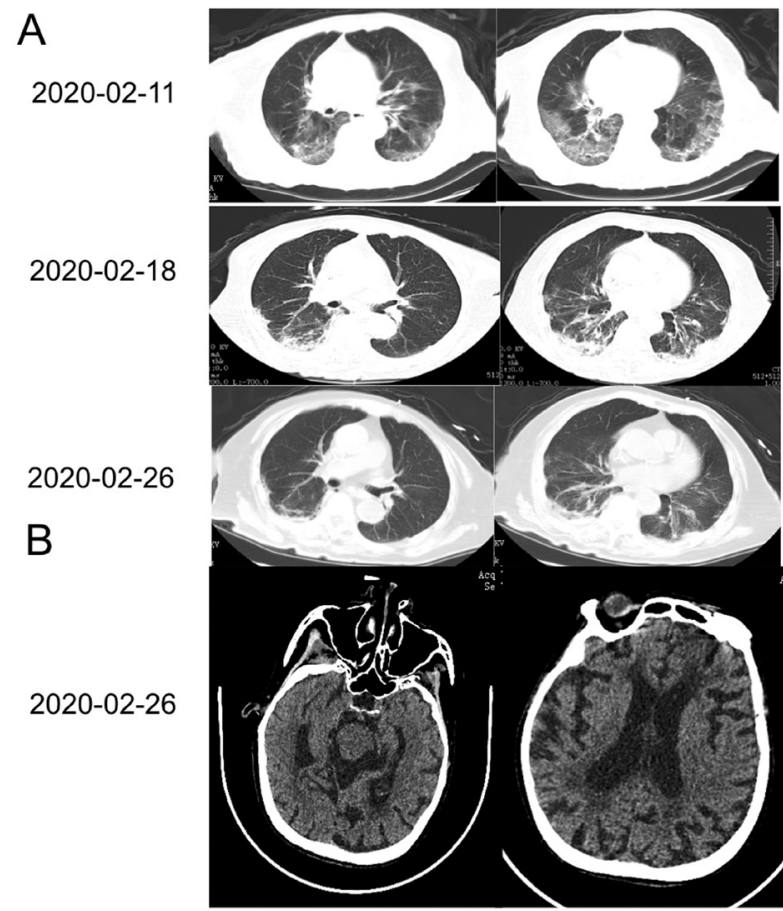

Figure 1. Representative CT and MRI images of the patient. (A) Chest CT images revealed bilateral GGO and segmental areas of consolidation at 11 days after disease onset. These GGOs were gradually resolved by days 18 and 26 after disease. (B) The head CT on the day that the patient entered the coma, which did not present any remarkable findings. GGO, ground-glass opacity. hematogenous spread from the systemic circulation to the cerebral circulation, and via dissemination through the cribriform plate and olfactory bulb (26). Furthermore, SARS-CoV-2 was reported to enter the CNS via binding to angiotensin converting enzyme 2 expressed in the capillary endothelium of the blood-brain barrier (27). SARS-CoV-2 RNA was detected in the CSF of 3 patients with COVID-19 reported in the literature review, of these 3 patients, 2 patients presented with abnormal neuroimaging findings. SARS-CoV-2 may also reach the CNS via trans-synaptic propagation through the nasal cavity. This neuronal approach is consistent with the clinical observation that certain patients with COVID-19 develop anosmia (28). However, there was no evidence of anosmia/dysgeusia in the cases reviewed.

Certain cases included in the present literature review were in a hyperinflammatory state secondary to SARS-CoV-2 infection, with massive release of cytokines and chemokines. Patient 2 in Table II and another 2 previously reported cases (29) had obvious elevation of CSF cytokines accompanied by exacerbation of neurological symptoms. Acute necrotizing encephalopathy was also recently reported in patients with COVID-19 (30). Thus, a hyperinflammatory state secondary to infection may have an important role in CNS injury by SARS-CoV-2.

The present analysis indicated that SARS-CoV-2 infected meningitis/encephalitis had a relatively non-fatal process with complete clinical recovery in the majority of cases. Specifically, almost $60 \%$ of patients exhibited neurological recovery, but with certain symptoms remaining, including 


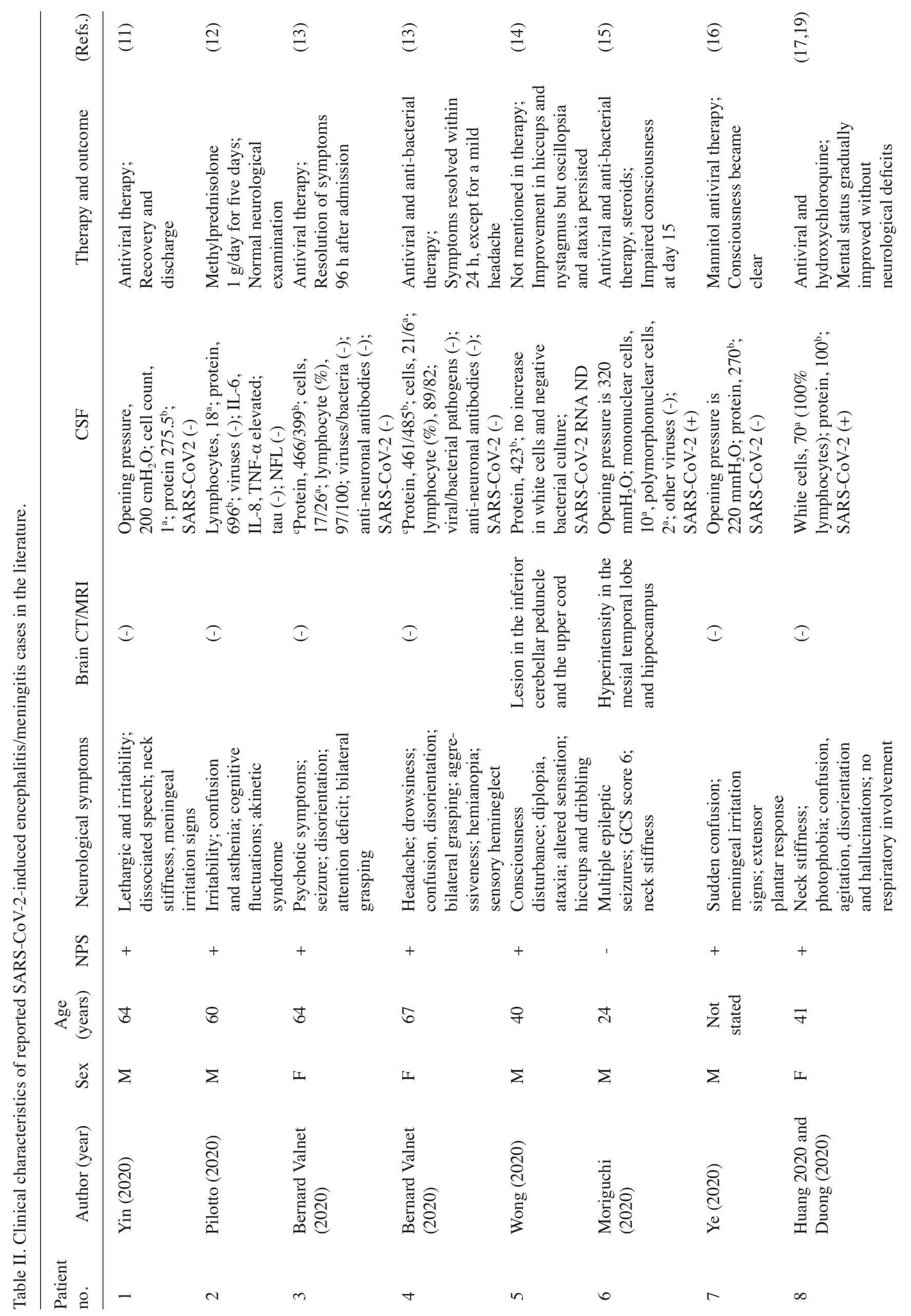




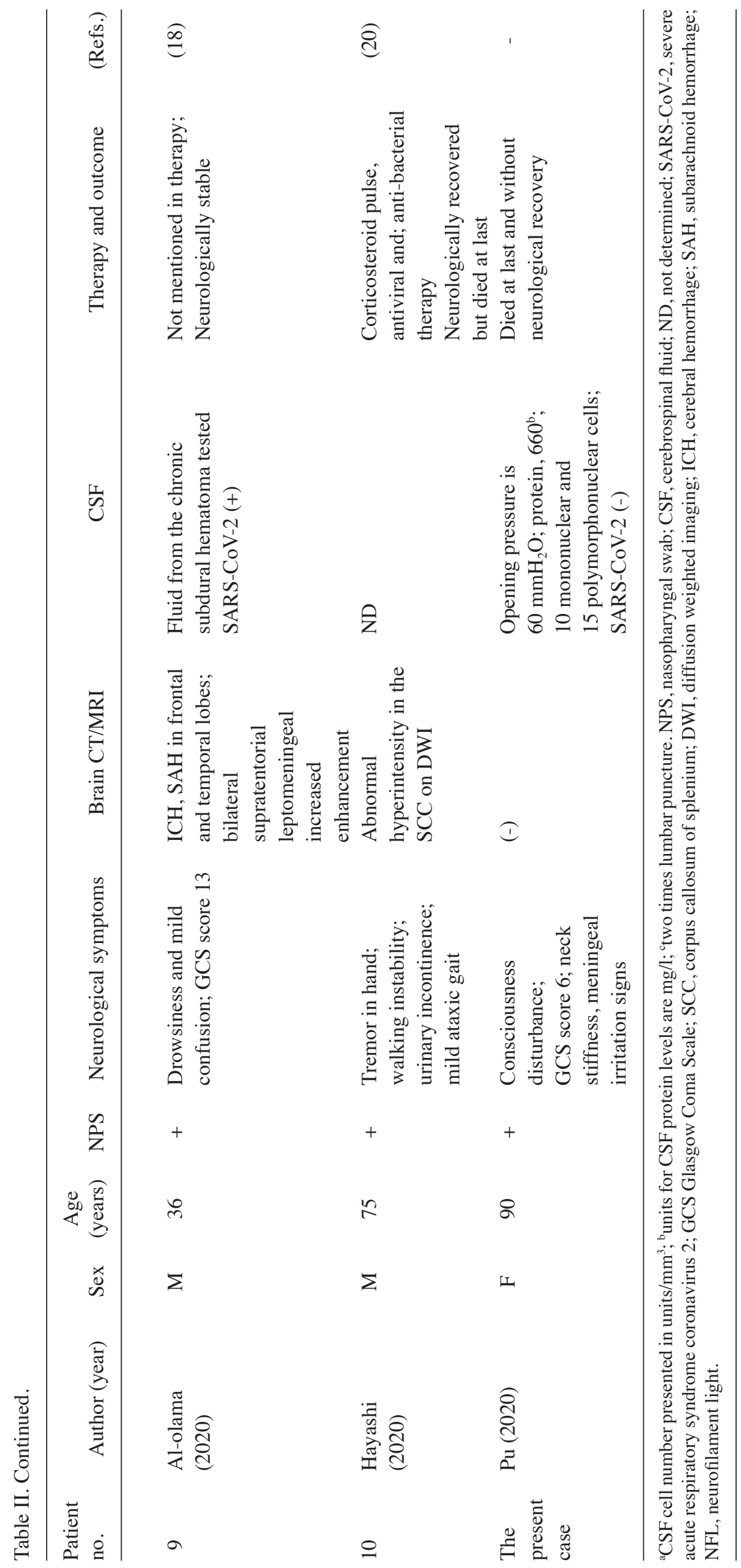


headache. This suggests that SARS-CoV-2 may induce a viral encephalitis or aseptic meningitis. With viral clearance and use of corticosteroids, the CSF pressure was gradually reduced and the neurological manifestations gradually improved. Immunoinhibition therapy is effective for reducing CSF cytokines and associated neurological manifestations. The present analysis suggested a role for cytokine-mediated neuroinflammation in these patients. Of note, senior patients (>60 years old), those positive for SARS-CoV-2 RNA in the CSF and those with brain MRI abnormalities tended to have poor outcomes.

In conclusion, COVID-19 infection may be associated with meningitis/encephalitis. The initial symptoms vary, although changes in consciousness, seizures and meningeal irritation signs were most frequent. Furthermore, CSF protein and white cell levels were typically elevated, with positive SARS-CoV RNA and elevated cytokine levels in the CSF in certain patients. Of note, most cases had favorable outcomes, except for older patients. A limitation of the present study was the lack of brain MRI in certain patients, including the present case. However, MRI was difficult to perform in certain patients due to medical isolation during the COVID-19 pandemic. Another limitation was that inflammatory cytokines in the CSF were not examined in the present case, which would have been required to confirm the disease pathogenesis.

\section{Acknowledgements}

Not applicable.

\section{Funding}

The present study was supported by the China-Japan Friendship Hospital funding for Youth (grant no. 2019-1-QN-23 and 2018-2-QN-34).

\section{Availability of data and materials}

The datasets used and/or analyzed during the current study are available from the corresponding author on reasonable request.

\section{Authors' contributions}

PL and XPW contributed to the conception and design of the study. FP contributed to the acquisition and analyses of data. PL performed the literature review. YZ, LZ, LS, YW, NL, PH and $\mathrm{TH}$ contributed to analyzing and drafting the manuscript, PL, YZ, LZ and LS contributed to preparing the figures. PL and XPW check and confirm the raw data of the study. All authors read and approved the final version of the manuscript.

\section{Ethics approval and consent to participate}

The study was approved by the ethics committee of the China-Japan Friendship Hospital (Beijing, China).

\section{Patient consent for publication}

The patient's guardians provided informed written consent for the case report.

\section{Competing interests}

The authors declare that they have no competing interests.

\section{References}

1. Marini JJ and Gattinoni L: Management of COVID-19 respiratory distress. JAMA 323: 2329-2330, 2020.

2. The Lancet Neurology: The neurological impact of COVID-19. Lancet Neurol 19: 471, 2020.

3. Favas TT, Dev P, Chaurasia RN, Chakravarty K, Mishra R, Joshi D, Mishra VN, Kumar A, Singh VK, Pandey M and Pathak A: Neurological manifestations of COVID-19: A systematic review and meta-analysis of proportions. Neurol Sci 41: 3437-3470, 2020.

4. Harapan BN and Yoo HJ: Neurological symptoms, manifestations, and complications associated with severe acute respiratory syndrome coronavirus 2 (SARS-CoV-2) and coronavirus disease 19 (COVID-19). J Neurol: Jan 21, 2021 (Epub ahead of print).

5. Lesser IA and Nienhuis CP: The impact of COVID-19 on physical activity behavior and well-being of Canadians. Int J Environ Res Public Health 17: 3899, 2020.

6. Nunnari G, Sanfilippo C, Castrogiovanni P, Imbesi R, Li Volti G, Barbagallo I, Musumeci G and Di Rosa M: Network perturbation analysis in human bronchial epithelial cells following SARS-CoV2 infection. Exp Cell Res 395: 112204, 2020.

7. Zhou Z, Kang H, Li S and Zhao X: Understanding the neurotropic characteristics of SARS-CoV-2: From neurological manifestations of COVID-19 to potential neurotropic mechanisms. J Neurol 267: 2179-2184, 2020.

8. Lechien JR, Barillari MR, Jouffe L and Saussez S: Anosmia is a key symptom of COVID-19 infection and should be used as a diagnostic tool. Ear Nose Throat J 99: 577-578, 2020.

9. Chiu A, Fischbein N, Wintermark M, Zaharchuk G, Yun PT and Zeineh M: COVID-19-induced anosmia associated with olfactory bulb atrophy. Neuroradiology 63: 147-148, 2021.

10. Altundag A, Saatci O, Sanli DET, Duz OA, Sanli AN, Olmuscelik O, Temirbekov D, Kandemirli SG and Karaaltin AB: The temporal course of COVID-19 anosmia and relation to other clinical symptoms. Eur Arch Otorhinolaryngol: Nov 25, 2020 (Epub ahead of print).

11. Yin R, Feng W, Wang T, Chen G, Wu T, Chen D, Lv T and Xiang D: Concomitant neurological symptoms observed in a patient diagnosed with coronavirus disease 2019. J Med Virol 92: 1782-1784, 2020

12. Pilotto A, Odolini S, Masciocchi S, Comelli A, Volonghi I, Gazzina S, Nocivelli S, Pezzini A, Focà E, Caruso A, et al: Steroid-responsive encephalitis in coronavirus disease 2019. Ann Neurol 88: 423-427, 2020.

13. Bernard Valnet R, Pizzarotti B, Anichini A, Demars Y, Russo E, Schmidhauser M, Cerutti-Sola J, Rossetti AO and Du Pasquier R: Two patients with acute meningoencephalitis concomitant with SARS-CoV-2 infection. Eur J Neurol 27: e43-e44, 2020.

14. Wong PF, Craik S, Newman P, Makan A, Srinivasan K, Crawford E, Dev D, Moudgil H and Ahmad N: Lessons of the month 1: A case of rhombencephalitis as a rare complication of acute COVID-19 infection. Clin Med (Lond) 20: 293-294, 2020.

15. Moriguchi T, Harii N, Goto J, Harada D, Sugawara H, Takamino J, Ueno M, Sakata H, Kondo K, Myose N, et al: A first case of meningitis/encephalitis associated with SARS-Coronavirus-2. Int J Infect Dis 94: 55-58, 2020.

16. Ye M, Ren Y and Lv T: Encephalitis as a clinical manifestation of COVID-19. Brain Behav Immun 88: 945-946, 2020.

17. Huang YH, Jiang D and Huang JT: SARS-CoV-2 detected in cerebrospinal fluid by PCR in a case of COVID-19 encephalitis. Brain Behav Immun 87: 149, 2020.

18. Al-Olama M, Rashid A and Garozzo D: COVID-19-associated meningoencephalitis complicated with intracranial hemorrhage: A case report. Acta Neurochir (Wien) 162: 1495-1499, 2020.

19. Duong L, Xu P and Liu A: Meningoencephalitis without respiratory failure in a young female patient with COVID-19 infection in downtown Los Angeles, early April 2020. Brain Behav Immun 87: 33, 2020.

20. Hayashi M, Sahashi Y, Baba Y, Okura H and Shimohata T: COVID-19-associated mild encephalitis/encephalopathy with a reversible splenial lesion. J Neurol Sci 415: 116941, 2020. 
21. Lovati C, Osio M and Pantoni L: Diagnosing herpes simplex-1 encephalitis at the time of COVID-19 pandemic. Neurol Sci 41: 1361-1364, 2020.

22. Zayet S, Ben Abdallah Y, Royer PY, Toko L, Gendrin V and Klopfenstein T: Encephalopathy in patients with COVID-19: 'Causality or coincidence?'. J Med Virol 93: 1193, 2020.

23. Dogan L, Kaya D, Sarikaya T, Zengin R, Dincer A, Akinci IO and Afsar N: Plasmapheresis treatment in COVID-19-related autoimmune meningoencephalitis: Case series. Brain Behav Immun 87: 155-158, 2020.

24. Chen CJ, Hsieh LL, Lin SK, Wang CF, Huang YH, Lin SY and Lu PL: Optimization of the CDC protocol of molecular diagnosis of COVID-19 for timely diagnosis. Diagnostics (Basel) 10: 333, 2020.

25. Ellul MA, Benjamin L, Singh B, Lant S, Michael BD Easton A, Kneen R, Defres S, Sejvar J and Solomon T: Neurological associations of COVID-19. Lancet Neurol 19: 767-783, 2020.
26. Montalvan V,Lee J, Bueso T, De Toledo J and Rivas K: Neurological manifestations of COVID-19 and other coronavirus infections: A systematic review. Clin Neurol Neurosurg 194: 105921, 2020.

27. Khaleeq A, Ali U and Syeda H: Evidence of the COVID-19 virus targeting the CNS: Tissue distribution, host-virus interaction, and proposed neurotropic mechanisms. ACS Chem Neuroscience 11: 995-998, 2020

28. Guan WJ, Ni ZY, Hu Y, Liang WH, Ou CQ, He JX, Liu L, Shan H, Lei CL, Hui DSC, et al: Clinical characteristics of coronavirus disease 2019 in China. N Engl J Med 382: 1708-1720, 2020.

29. Bodro M, Compta Y, Llansó L, Esteller D, Doncel-Moriano A, Mesa A, Rodríguez A, Sarto J, Martínez-Hernandez E, Vlagea A, et al: Increased CSF levels of IL-1 $\beta$, IL-6, and ACE in SARS-CoV-2-associated encephalitis. Neurol Neuroimmunol Neuroinflamm 7: e821, 2020.

30. Elkady A and Rabinstein AA: Acute necrotizing encephalopathy and myocarditis in a young patient with COVID-19. Neurol Neuroimmunol Neuroinflamm 7: e801, 2020. 